\author{
Jen-San Chen \\ Professor \\ Kwin-Lin Chen \\ Graduate Student \\ Department of Mechanical Engineering, \\ National Taiwan University, \\ Taipei, Taiwan 10617
}

\title{
The Role of Lagrangian Strain in the Dynamic Response of a Flexible Connecting Rod
}

Previous researches on the dynamic response of a flexible connecting rod can be categorized by the ways the axial load in the rod is being formulated. The axial load may be assumed to be (1) dependent only on time and can be obtained by treating the rod as rigid, (2) related to the transverse displacement by integrating the axial equilibrium equation, and (3) proportional to linear strain. This paper examines the validity of these formulations by first deriving the equations of motion assuming the axial load to be proportional to the Lagrangian strain. In order for the dimensionless displacements to be in the order of $O(1)$, different nondimensionalization schemes have to be adopted for low and high crank speeds. The slenderness ratio of the connecting rod arises naturally as a small parameter with which the order of magnitude of each term in the equations of motion, and the implication of these simplified formulations can be examined. It is found that the formulations in previous researches give satisfactory results only when the crank speed is low. On the other hand when the crank speed is comparable to the first bending natural frequency of the connecting rod, these simplified formulations overestimate considerably the dynamic response because terms of significant order of magnitude are removed inadequately. [DOI: 10.1115/1.1415738]

\section{Introduction}

Dynamic analysis of flexible mechanisms has become an important research topic in recent years, partly due to the increasing precision demand in machines operating at high speed. The subjects of interest include transient response, steady state response, and dynamic stability analysis. In general the mathematical complexity involved in the analysis is so overwhelming that finite element method may be the only feasible technique to attack the problems [1-3]. However considerable insight may be obtained by focusing on the detailed mathematical investigation of certain mechanism which retains the important characteristics of dynamic vibration while renders itself to solvability. The simplest mechanism of such kind is a slider-crank mechanism with elastic connecting rod.

The flexible connecting rod can be considered as a beam undergoing large rigid body motion and may deform in both the axial and transverse directions. A complete formulation must take into account the coupling effect of the axial and transverse vibrations. Due to the inherent mathematical complexity, it was very common in the past to adopt various assumptions in the formulation to simplify the solution procedure. It is important to verify the validity of these assumptions with more accurate formulation. In the following, we categorize the common simplifications adopted in the literature based on the ways the axial load in the rod is being formulated.

1 Assume that the axial force is a function of time only and can be obtained by assuming that the connecting rod is rigid. The resulted equation of motion in terms of the transverse displacement is an inhomogeneous Mathieu equation. Neubauer et al. [4] adopted this simplest approach to investigate the effects of crank speed, length ratio between crank arm and connecting rod, and slider mass on the dynamic response of the connecting rod. The solutions from normal-mode expansion and from finite difference method are compared. Badlani and Midha [5,6] also adopted this approach to investigate the dynamic response of a connecting rod

Contributed by the Design Automation Committee for publication in the JOURNAL OF MECHANICAL DESIGN. Manuscript received May 1999. Associate Editor: H. Lankarani. with initial curvature and material damping. Both Euler beam and Timoshenko beam are considered by Badlani and Kleinhenz [7]. Tadjbakhsh [8] first formulated the equations of motion for a general mechanism and later focused on a slider-crank mechanism. He used Floquet theory to determine the critical values of various dimensionless parameters which may cause instability of the mechanism, see also [9]. Zhu and Chen [10] used a perturbation technique to study the dynamic stability of the connecting rod.

2 Relate the axial force to the transverse displacement by integrating the axial equilibrium equation after neglecting the axial vibration and the relative centrifugal force. This formulation is an improvement of formulation (1), and has been adopted by Viscomi and Ayre [11], and Hsieh and Shaw [12]. Hsieh and Shaw [12] also investigated the foreshortening effect on the parametric resonance of the mechanism.

3 Consider the effect of axial vibration, but assume that the axial force is proportional to the linear axial strain. The two equations of motion involve both the axial and transverse displacements. Jasinski et al. $[13,14]$ adopted this approach but "linearized" the equations of motion by deleting all the terms involving product of displacements. As a consequence the stiffening effect of the axial load is missing. Chu and Pan [15] also adopted this approach but used inadequate shape function for the axial displacement in their Galerkin's discretization procedure. As a consequence the stiffening effect of the axial load was also removed. These two papers did not consider the inertia of the slider. Fung and Chen [16] considered the steady state response of a Timoshenko beam with time dependent boundary conditions based on a finite element formulation. They also "linearized" the equations of motion by dropping all the terms containing products of displacements.

Although these above simplifications reduce the complexity of the solution procedure considerably, it has never been examined in the literature to what extent these assumptions are valid. One obvious problem with the linear strain formulation, for instance, is when the crank rotates at a speed comparable to the bending natural frequency, it is reasonable to argue that the quadratic component in the axial Lagrangian strain may not be negligible any more. In the present paper we start the analysis by adopting the 
most rigorous approach assuming that the axial force is proportional to the Lagrangian axial strain, which involves both the axial and transverse displacements. The equations of motion are then nondimensionalized by assuming that the dimensionless displacements are of order $\mathrm{O}(1)$. This assumption is checked later by numerical simulation. The slenderness ratio of the connecting rod arises automatically as a small parameter and can be used to determine the relative significance of each term in the equations of motion. It is found that the nondimensionalization schemes ought to be different for the high and low crank speeds. With the appropriate dimensionless equations of motion for different crank speeds, we can then simplify the equations of motion by ignoring those terms with smaller order of magnitude. It is found that the three simplifications give similar result as the Lagrangian strain formulation does when the crank speed is low. On the other hand they induce considerable errors when the crank speed is comparable to the first bending natural frequency of the connecting rod because terms of significant order of magnitude are ignored inadequately.

\section{Equations of Motion}

Figure 1 shows a slider-crank mechanism. The rigid crank with length $a$ rotates with constant speed $\Omega$. The length, cross section area, mass density, and Young's modulus of the elastic connecting rod are $L, A, \rho$, and $E$, respectively. The mass of the slider is $m_{s}$. $X O Y$ is an inertial frame with its origin attached at the center of the rotating crank. $x A y$ is a moving frame with $x$-axis passing through the two ends of the connecting rod. $u(x, t)$ and $v(x, t)$ denote the axial and transverse displacements of the connecting rod. From the force balance in the axial and transverse directions and the moment balance of a small element of the connecting rod, we obtain the three equilibrium Eqs. [7],

$$
\begin{gathered}
P_{, x}=\rho A\left[u_{, t t}-2 v_{, t} \dot{\phi}-(x+u) \dot{\phi}^{2}-v \ddot{\phi}-a \Omega^{2} \cos (\Omega t-\phi)\right] \\
Q_{, x}=\rho A\left[v_{, t t}+2 u_{, t} \dot{\phi}-v \dot{\phi}^{2}+(x+u) \ddot{\phi}-a \Omega^{2} \sin (\Omega t-\phi)\right] \\
M_{, x}+Q-P v_{, x}=0
\end{gathered}
$$

$P, Q$, and $M$ are the axial force, shear force, and bending moment, respectively, at position $x$ on the connecting rod. $\phi$ is the angle between the $x$-axis and $X$-axis, and can be obtained from rigid body kinematics,

$$
\phi=\sin ^{-1}\left(-\frac{a \sin \Omega t}{L}\right)
$$

The constitutive laws for an Euler beam are used,

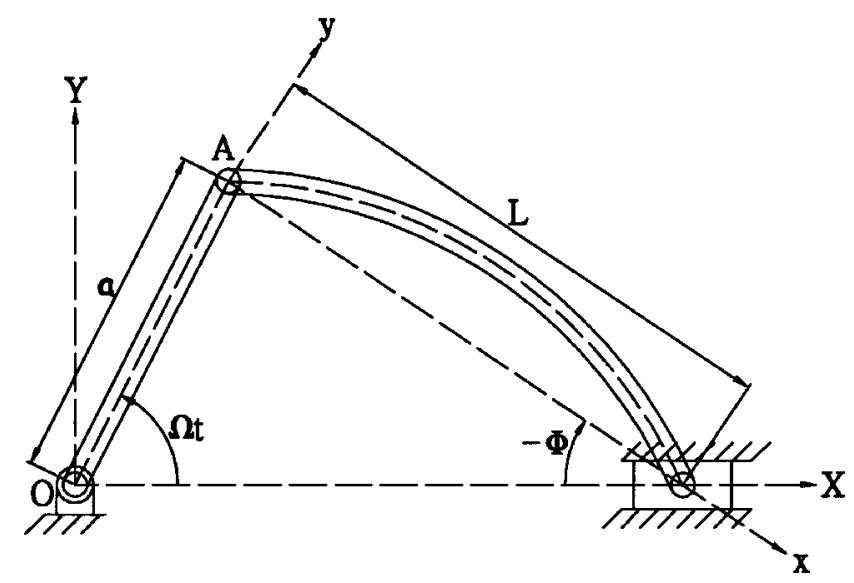

Fig. 1 Schematic diagram of a slider and crank mechanism

$$
\begin{gathered}
P=E A\left(u_{, x}+\frac{1}{2} v_{, x}^{2}\right) \\
M=E I v_{, x x}
\end{gathered}
$$

$I$ is the area moment of inertia of the connecting rod,

$$
I=A r^{2}
$$

where $r$ is the radius of gyration of the cross section. By substituting Eqs. (3),(5),(6) into Eqs. (1),(2) we obtain the following equations of motion,

$$
\begin{aligned}
& u_{, t t}-\dot{\phi}^{2} u- \frac{E}{\rho}\left(u_{, x x}+v_{, x} v_{, x x}\right)-2 \dot{\phi} v_{, t}-\ddot{\phi} v-x \dot{\phi}^{2} \\
&-a \Omega^{2} \cos (\Omega t-\phi)=0 \\
& v_{, t t}-\dot{\phi}^{2} v+\frac{E I}{\rho A} v_{, x x x x}+2 \dot{\phi} u_{, t}+\ddot{\phi} u \\
&-\frac{E}{\rho}\left(u_{, x x} v_{, x}+u_{, x} v_{, x x}+\frac{3}{2} v_{, x}^{2} v_{, x x}\right) \\
&+x \ddot{\phi}-a \Omega^{2} \sin (\Omega t-\phi)=0
\end{aligned}
$$

The connecting rod is assumed to be simply-supported at two ends. Therefore the boundary conditions for $u$ and $v$ are

$$
v(0, t)=v(L, t)=v_{, x x}(0, t)=v_{, x x}(L, t)=u(0, t)=0
$$

From the force balance of the slider we can obtain the boundary condition for $u$ at $x=L$,

$$
\begin{aligned}
u_{, x}+\left.\frac{1}{2} v_{, x}^{2}\right|_{x=L}= & -\frac{1}{E A \cos \phi}\left[-\frac{1}{3} \rho A L^{2} \ddot{\phi} \sin \phi\right. \\
& +\frac{1}{2} \rho A L a \Omega^{2} \sin \phi \sin (\Omega t-\phi) \\
& \left.+m_{s}\left(-a \Omega^{2} \cos \Omega t-L \dot{\phi}^{2} \cos \phi+a \ddot{\phi} \sin \Omega t\right)\right]
\end{aligned}
$$

\section{Low Crank Speed}

We now investigate the case when the crank rotates at low speed compared to the first bending natural frequency of the connecting rod. Equations (7) and (8) can be nondimensionalized by introducing the following dimensionless quantities,

$$
\begin{gathered}
x^{*}=\frac{x}{L}, \quad a^{*}=\frac{a}{L}, \quad u^{*}=\frac{L^{2}}{r^{3}} u, \quad v^{*}=\frac{L v}{r^{2}}, \quad \varepsilon=\frac{r}{L}, \\
m_{s}^{*}=\frac{m_{s}}{\rho A L}, \quad P^{*}=\frac{P}{E A}, \quad \Omega^{*}=\frac{\Omega}{\omega_{b}}, \quad t^{*}=\omega_{b} t
\end{gathered}
$$

$\omega_{b}$ is the lowest bending natural frequency of the connecting rod,

$$
\omega_{b}=\frac{\pi^{2}}{L^{2}} \sqrt{\frac{E I}{\rho A}}
$$

The ratio between the bending natural frequency and the longitudinal natural frequency of the connecting rod is proportional to $\varepsilon$, the slenderness ratio parameter. In the above nondimensionalization we have assumed that both the dimensionless displacements $u^{*}$ and $v^{*}$ are of the order $\mathrm{O}(1)$. Whether this assumption is valid can be verified by numerical simulation later. After substituting these relations into Eqs. (7), (8), and (10), and dropping the superposed asterisks for simplicity, we can rewrite the equations of motion and the inhomogeneous boundary condition in the following forms, 


$$
\begin{gathered}
\varepsilon^{3}\left(u_{, t t}-\dot{\phi}^{2} u\right)-\varepsilon^{2}\left(2 \dot{\phi}_{, t}+\ddot{\phi} v+\frac{1}{\pi^{4}} v_{, x} v_{, x x}\right) \\
-\varepsilon \frac{1}{\pi^{4}} u_{, x x}-x \dot{\phi}^{2}-a \Omega^{2} \cos (\Omega t-\phi)=0 \\
-\frac{3 \varepsilon^{4}}{2 \pi^{4}} v_{, x}^{2} v_{, x x}+\varepsilon^{3}\left[2 \dot{\phi} u_{, t}+\ddot{\phi} u-\frac{1}{\pi^{4}}\left(u_{, x x} v_{, x}+u_{, x} v_{, x x}\right)\right] \\
+\varepsilon^{2}\left(v_{, t t}-\dot{\phi}^{2} v+\frac{1}{\pi^{4}} v_{, x x x x}\right)+x \ddot{\phi}-a \Omega^{2} \sin (\Omega t-\phi)=0
\end{gathered}
$$

$$
\frac{P(1, t)}{\varepsilon^{2}}=\varepsilon u_{, x}+\left.\varepsilon^{2} \frac{1}{2} v_{, x}^{2}\right|_{x=1}=-\frac{\pi^{4}}{\cos \phi}\left(\Theta-m_{s} a \Omega^{2} \cos \Omega t\right)
$$

where

$$
\begin{aligned}
\Theta= & \frac{a}{3} \ddot{\phi} \sin \Omega t-\frac{1}{2} a^{2} \Omega^{2} \sin \Omega t \sin (\Omega t-\phi) \\
& +m_{s}\left(-\dot{\phi}^{2} \cos \phi+a \ddot{\phi} \sin \Omega t\right)
\end{aligned}
$$

We assume a one-mode approximation for $u$ and $v$ as following

$$
\begin{aligned}
& u(x, t)=f(t) \sin \frac{\pi x}{2} \\
& v(x, t)=g(t) \sin \pi x
\end{aligned}
$$

After substituting Eqs. (14) and (15) into Eqs. (11) and (12), multiplying Eqs. (11) and (12) by $\sin (\pi x / 2)$ and $\sin \pi x$, respectively, and then integrating by parts from $x=0$ to 1 we obtain

$$
\begin{aligned}
\varepsilon^{3}\left(\ddot{f}-\dot{\phi}^{2} f\right)-\varepsilon^{2}\left[\frac{8}{3 \pi}(2 \dot{\phi} \dot{g}+\ddot{\phi} g)-\frac{7}{15 \pi^{2}} g^{2}\right]+\frac{\varepsilon}{4 \pi^{2}} f \\
=\frac{8}{\pi^{2}} \dot{\phi}^{2}+\frac{4}{\pi} a \Omega^{2} \cos (\Omega t-\phi) \\
\quad-\frac{2}{\cos \phi}\left(\Theta-m_{s} a \Omega^{2} \cos \Omega t\right) \\
\frac{3 \varepsilon^{4}}{8} g^{3}+\varepsilon^{3}\left[\frac{8}{3 \pi}(2 \dot{\phi} \dot{f}+\ddot{\phi} f)+\frac{14}{15 \pi^{2}} f g\right]+\varepsilon^{2}\left(\ddot{g}-\dot{\phi}^{2} g+g\right) \\
=-\frac{2}{\pi} \ddot{\phi}+\frac{4}{\pi} a \Omega^{2} \sin (\Omega t-\phi)
\end{aligned}
$$

By specifying initial conditions, Eqs. (16) and (17) can be solved by Runge-Kutta method. In the following we compare the common simplifications adopted in the literature with Eqs. (16) and (17).

1. Linear Strain Simplification. If we start the analysis by assuming linear strain in Eq. (5), then we have to modify Eq. (11) by deleting the term containing $\varepsilon^{2} v_{, x} v_{, x x}$, and modify (12) by deleting the term containing $\varepsilon^{4} v_{, x}^{2} v_{, x x}$. As a consequence, Eqs. (16) and (17) should be modified by dropping the terms containing $\varepsilon^{2} g^{2}$ and $\varepsilon^{4} g^{3}$, respectively, i.e.,

$$
\begin{gathered}
\varepsilon^{3}\left(\ddot{f}-\dot{\phi}^{2} f\right)-\varepsilon^{2} \frac{8}{3 \pi}(2 \dot{\phi} \dot{g}+\ddot{\phi} g)+\frac{\varepsilon}{4 \pi^{2}} f \\
=\frac{8}{\pi^{2}} \dot{\phi}^{2}+\frac{4}{\pi} a \Omega^{2} \cos (\Omega t-\phi) \\
-\frac{2}{\cos \phi}\left(\Theta-m_{s} a \Omega^{2} \cos \Omega t\right)
\end{gathered}
$$

$$
\begin{gathered}
\varepsilon^{3}\left[\frac{8}{3 \pi}(2 \dot{\phi} \dot{f}+\ddot{\phi} f)+\frac{14}{15 \pi^{2}} f g\right]+\varepsilon^{2}\left(\ddot{g}-\dot{\phi}^{2} g+g\right) \\
=-\frac{2}{\pi} \ddot{\phi}+\frac{4}{\pi} a \Omega^{2} \sin (\Omega t-\phi)
\end{gathered}
$$

Since these ignored terms are small in order of magnitude compared to other dominant terms, we expect that at low speed range, the linear strain assumption is a good approximation when the slenderness ratio $\varepsilon$ is small.

2. Axial Load by Integrating Axial Equilibrium Equation. In this approach we rewrite Eq. (11) by ignoring the $\varepsilon^{3}$-terms,

$$
P_{, x}=-\varepsilon^{2} \pi^{4}\left[\varepsilon^{2}\left(2 \dot{\phi} v_{, t}-\ddot{\phi} v\right)+x \dot{\phi}^{2}+a \Omega^{2} \cos (\Omega t-\phi)\right]
$$

The axial load $P$ can be integrated to

$$
\begin{aligned}
P(x, t)= & P(1, t)-\int_{1}^{x} \varepsilon^{2} \pi^{4}\left[\varepsilon^{2}\left(2 \dot{\phi} v_{, t}-\ddot{\phi} v\right)\right. \\
& \left.+\xi \dot{\phi}^{2}+a \Omega^{2} \cos (\Omega t-\phi)\right] d \xi
\end{aligned}
$$

$P(1, t)$ has been given in Eq. (13). Equation (21) can then be substituted into Eq. (12) to obtain

$$
\begin{aligned}
& -\frac{1}{\pi^{4}}\left(P v_{, x}\right)_{, x}+\varepsilon^{3}\left[2 \dot{\phi} u_{, t}+\ddot{\phi} u\right]+\varepsilon^{2}\left(v_{, t t}-\dot{\phi}^{2} v+\frac{1}{\pi^{4}} v_{, x x x x}\right) \\
& +x \ddot{\phi}-a \Omega^{2} \sin (\Omega t-\phi)=0
\end{aligned}
$$

Equation (22) is similar to the equation used by Viscomi and Ayre [11], and can be discretized by the one-mode approximation, Eq. (15), to

$$
\begin{aligned}
\varepsilon^{2}(\ddot{g} & \left.+\frac{m_{s} a \Omega^{2} \pi^{2}}{\cos \phi} \cos \Omega t g+g\right)+\varepsilon^{2} g\left[\left(-\frac{5}{4}+\frac{\pi^{2}}{3}\right) \dot{\phi}^{2}\right. \\
& \left.+\frac{\pi^{2}}{2} a \Omega^{2} \cos (\Omega t-\phi)-\frac{\pi^{2} \Theta}{\cos \phi}\right]+\varepsilon^{4} \pi\left(\ddot{\phi} g^{2}+2 \dot{\phi} g \dot{g}\right) \\
& =-\frac{2}{\pi} \ddot{\phi}+\frac{4}{\pi} a \Omega^{2} \sin (\Omega t-\phi)
\end{aligned}
$$

It is noted that Eq. (23) can also be obtained by expressing $f$ in terms of $g$ after ignoring the $\varepsilon^{3}$-terms in Eq. (18), and then substituting this expression into Eq. (19). The resulted single equation in terms of $g$ is very similar to Eq. (23) except some minor differences on the coefficients due to slightly different Galerkin's procedure. Since Eq. (23) is different from Eq. (18) only in the high order $\varepsilon^{3}$-terms, we expect this to be a good approximation to the linear strain formulation.

3. Time-Dependent-Only Axial Load Simplification. In this approach we further neglect the right hand side of the axial equilibrium Eq. (20) to obtain

$$
P_{, x}=0
$$

This dramatic simplification may be justified by assuming the crank-to-rod ratio $a$ to be small. The angle $\phi$ in Eq. (4) can then be approximated as

$$
\phi \approx-a \sin \Omega t
$$

By dropping the smaller terms containing $a^{2}$ in the inhomogeneous boundary condition (13) we obtain the axial load as a function of time only

$$
P(t)=\varepsilon^{2} \pi^{4} m_{s} a \Omega^{2} \cos \Omega t
$$

By dropping the terms $\varepsilon^{3}\left(2 \dot{\phi} u_{, t}+\ddot{\phi} u\right)$ and $\varepsilon^{2} \dot{\phi}^{2} v$ in Eq. (22) and making use of Eq. (25), we finally arrive at the single equation 


$$
\begin{array}{r}
\varepsilon^{2}\left(v_{, t t}+\frac{1}{\pi^{4}} v_{, x x x x}-m_{s} a \Omega^{2} \cos \Omega t v_{, x x}\right) \\
=(1-x) a \Omega^{2} \sin \Omega t+\frac{a^{2}}{2} \Omega^{2} \sin 2 \Omega t
\end{array}
$$

Equation (26) is the one used by Badlani and Midha [5]. After Galerkin's procedure, Eq. (26) can be discretized to

$$
\varepsilon^{2}\left(\ddot{g}+m_{s} a \Omega^{2} \pi^{2} \cos \Omega t g+g\right)=\frac{2 a \Omega^{2}}{\pi}(\sin \Omega t+a \sin 2 \Omega t)
$$

Equation (27) is a standard inhomogeneous Mathieu equation [17]. Equation (27) can also be obtained from Eq. (23) by ignoring the $\varepsilon^{4}$-terms and the terms inside the bracket.

Runge-Kutta Simulation. The responses $f$ and $g$ of the connecting rod at $\Omega=0.1$ based on the above four formulations can be calculated by Runge-Kutta method. The parameters used in the calculations are $a=0.1, \varepsilon=0.01, m_{s}=0.1$. The initial conditions are

$$
f(0)=\dot{f}(0)=g(0)=\dot{g}(0)=0
$$

The time step used in Runge-Kutta method is $10^{-3}$. Figure 2 shows the lateral response $g$ from $t=0$ to 200. The results from all four formulations are almost indistinguishable and we plot only the one from Lagrangian strain formulation as line (1) in Fig. 2. Figure 3 shows the response $f$ from $t=0$ to 1 . Again the results from linear and Lagrangian strain formulations are indistinguishable. In this small time span from $t=0$ to 1 , we observe only the free axial vibration component (homogeneous solution) of the response $f$. In order to observe the forced response component (particular solution) in the axial direction, we plot the envelop of response curve $f$ from $t=0$ to 200 in Fig. 4. Line (1) in Fig. 4 represents the envelope calculated from Lagrangian strain formulation. All other three simplified formulations give indistinguishable envelopes in Fig. 4 and are plotted as line (2). It is also important to observe that both $f$ and $g$ are of the order $\mathrm{O}(1)$, as was required in examining the order of magnitude of each term in the equations of motion.

As observed from Figs. 2 and 4 at low crank speed, both $f$ and $g$ contain two harmonic components, one corresponds to free vibration while the other corresponds to forced response. To predict these two components in closed forms we simplify Eqs. (16) and (17) by removing higher order terms in $\varepsilon$ and $a$ to

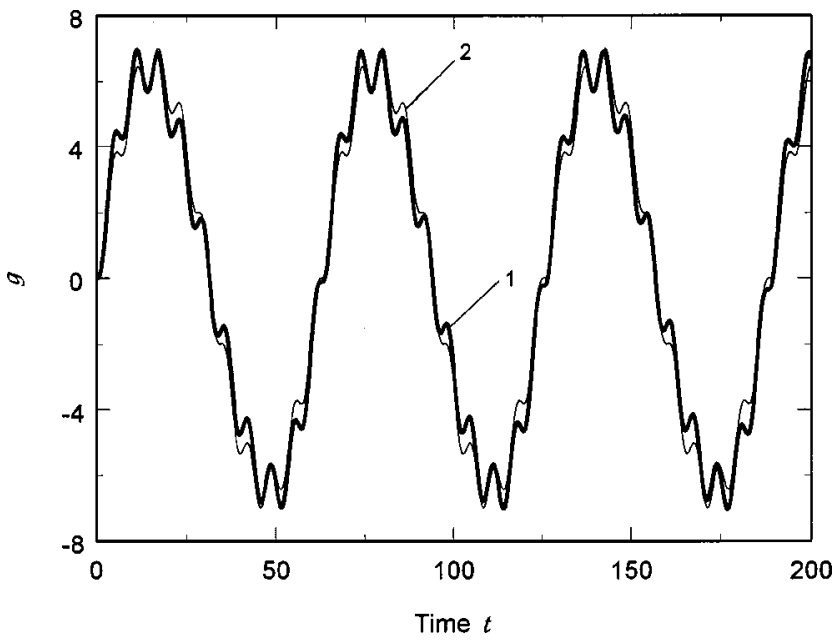

Fig. 2 Response $g$ of the connecting rod at $\Omega=0.1$. (1) Lagrangian strain formulation. (2) Solution predicted by Eq. (31).

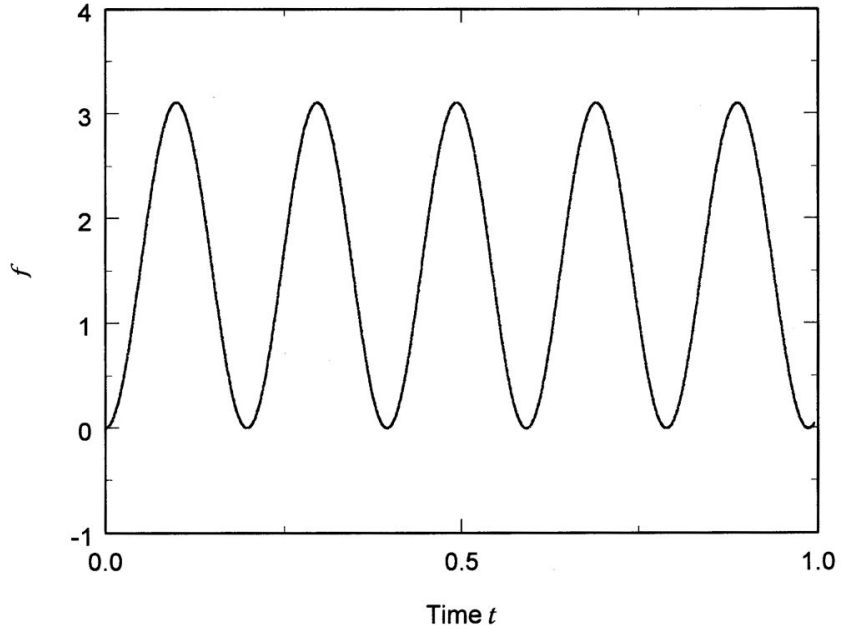

Fig. 3 Response $f$ of the connecting rod at $\Omega=0.1$

$$
\begin{gathered}
\varepsilon^{3} \ddot{f}+\frac{\varepsilon}{4 \pi^{2}} f=\left(2 m_{s}+\frac{4}{\pi}\right) a \Omega^{2} \cos \Omega t \\
\varepsilon^{2}(\ddot{g}+g)=\frac{2}{\pi} a \Omega^{2} \sin \Omega t
\end{gathered}
$$

After applying the initial conditions the solutions $f$ and $g$ of these two uncoupled equations are

$$
\begin{gathered}
f=\frac{4 \pi^{2} a \Omega^{2}}{\varepsilon\left(1-4 \varepsilon^{2} \pi^{2} \Omega^{2}\right)}\left(2 m_{s}+\frac{4}{\pi}\right)\left[\cos \Omega t-\cos \left(\frac{t}{2 \varepsilon \pi}\right)\right] \\
g=\frac{2 a \Omega^{2}}{\varepsilon^{2} \pi\left(1-\Omega^{2}\right)}(\sin \Omega t-\Omega \sin t)
\end{gathered}
$$

Solution (31) is plotted in Fig. 2 as line (2). The envelope of solution (30) is plotted in Fig. 4 as line (3). The fact that these two solutions are very good approximation to those from Lagrangian strain formulation confirms the general belief that at low crank speed the coupling effect between the axial and the transverse vibrations are negligible.

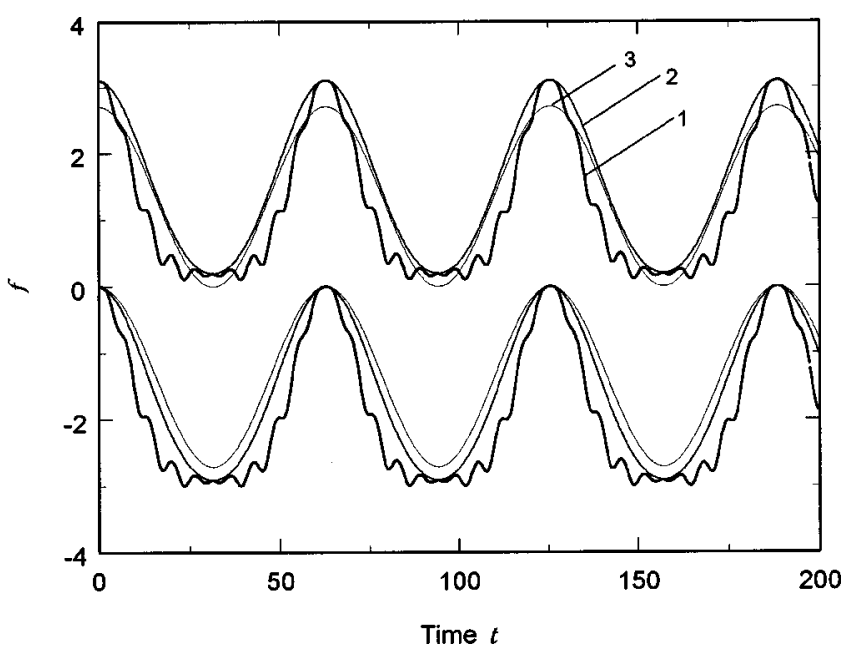

Fig. 4 Envelope of response $f$ of the connecting rod at $\Omega=0.1$. (1) Lagrangian strain formulation. (2) Linear strain formulation. (3) Solution predicted by Eq. (30). 


\section{High Crank Speed}

We now examine the case when the crank rotates at a speed comparable to the first bending natural frequency of the connecting rod. By expecting the displacements to be larger than those at low speed we redefine the dimensionless displacements as

$$
u^{*}=\frac{L}{r^{2}} u, \quad v^{*}=\frac{v}{r}
$$

The definitions of other parameters remain the same. Again, whether displacements $u^{*}$ and $v^{*}$ are of the order $\mathrm{O}(1)$ needs to be checked by numerical simulation later. The dimensionless equations of motion and the inhomogeneous boundary condition are rewritten in the following forms,

$$
\begin{gathered}
\varepsilon^{2}\left(u_{, t t}-\dot{\phi}^{2} u\right)-\varepsilon\left(2 \dot{\phi} v_{, t}+\ddot{\phi} v\right)-\frac{1}{\pi^{4}}\left(u_{, x x}+v_{, x} v_{, x x}\right) \\
-x \dot{\phi}^{2}-a \Omega^{2} \cos (\Omega t-\phi)=0 \\
\varepsilon^{2}\left(2 \dot{\phi} u_{, t}+\ddot{\phi} u\right)+\varepsilon\left[v_{, t t}-\dot{\phi}^{2} v+\frac{1}{\pi^{4}}\left(v_{, x x x x}-u_{, x x} v_{, x}-u_{, x} v_{, x x}\right.\right. \\
\left.\left.-\frac{3}{2} v_{, x}^{2} v_{, x x}\right)\right]+x \ddot{\phi}-a \Omega^{2} \sin (\Omega t-\phi)=0 \\
\frac{P(1, t)}{\varepsilon^{2}}=u_{, x}+\left.\frac{1}{2} v_{, x}^{2}\right|_{x=1}=-\frac{\pi^{4}}{\cos \phi}\left(\Theta-m_{s} a \Omega^{2} \cos \Omega t\right)
\end{gathered}
$$

We notice from Eq. (32) that the terms $v_{, x} v_{, x x}$ and $u_{, x x}$ are of the same order of magnitude and both are dominant terms at high crank speed. Therefore, it will be erroneous to delete a term containing product of displacements as in the conventional "linearization" procedure. The term $v_{, x} v_{, x x}$ is from the quadratic component in the Lagrangian strain, while the term $u_{, x x}$ is from the linear component in the strain. Similar observation on Eq. (33) also reveals that the contribution from the quadratic component in the Lagrangian strain is as significant as the one from the linear component at high crank speed.

Following the similar one-mode Galerkin's procedure, we obtain the discretized equations

$$
\begin{gathered}
\varepsilon^{2}\left(\ddot{f}-\dot{\phi}^{2} f\right)-\varepsilon \frac{8}{3 \pi}(2 \dot{\phi} \dot{g}+\ddot{\phi} g)+\frac{1}{\pi^{2}}\left(\frac{f}{4}+\frac{7}{15} g^{2}\right) \\
=\frac{8}{\pi^{2}} \dot{\phi}^{2}+\frac{4}{\pi} a \Omega^{2} \cos (\Omega t-\phi) \\
\quad-\frac{2}{\cos \phi}\left(\Theta-m_{s} a \Omega^{2} \cos \Omega t\right) \\
\varepsilon^{2} \frac{8}{3 \pi}(2 \dot{\phi} \dot{f}+\ddot{\phi} f)+\varepsilon\left(\ddot{g}-\dot{\phi}^{2} g+g+\frac{14}{15 \pi^{2}} f g+\frac{3}{8} g^{3}\right) \\
=-\frac{2}{\pi} \ddot{\phi}+\frac{4}{\pi} a \Omega^{2} \sin (\Omega t-\phi)
\end{gathered}
$$

1. Linear Strain Simplification. In the case if we start the analysis by assuming linear strain in Eq. (5) we have to modify Eq. (32) by deleting the term containing $v_{, x} v_{, x x}$, and modify (33) by deleting the term containing $\varepsilon v_{, x}^{2} v_{, x x}$. As a consequence, Eqs. (35) and (36) should be modified by dropping the terms containing $g^{2}$ and $\varepsilon g^{3}$, respectively. Since these terms are actually the dominant terms in the equations of motion, deleting them will induce significant errors. Therefore, we expect that linear strain assumption is a poor approximation at high crank speed.

2. Axial Load by Integrating Axial Equilibrium Equation. By following the similar procedure as in the low crank speed range, we arrive at the following equations

$$
\begin{gathered}
-\frac{1}{\varepsilon \pi^{4}}\left(P v_{, x}\right)_{, x}+\varepsilon^{2}\left[2 \dot{\phi} u_{, t}+\ddot{\phi} u\right]+\varepsilon\left(v_{, t t}-\dot{\phi}^{2} v+\frac{1}{\pi^{4}} v_{, x x x x}\right) \\
+x \ddot{\phi}-a \Omega^{2} \sin (\Omega t-\phi)=0 \\
\varepsilon\left(\ddot{g}+\frac{m_{s} a \Omega^{2} \pi^{2}}{\cos \phi} \cos \Omega t g+g\right)+\varepsilon g\left[\left(-\frac{5}{4}+\frac{\pi^{2}}{3}\right) \dot{\phi}^{2}\right. \\
\left.+\frac{\pi^{2}}{2} a \Omega^{2} \cos (\Omega t-\phi)-\frac{\pi^{2} \Theta}{\cos \phi}\right]+\varepsilon^{2} \pi\left(\ddot{\phi} g^{2}+2 \dot{\phi} g \dot{g}\right) \\
=-\frac{2}{\pi} \ddot{\phi}+\frac{4}{\pi} a \Omega^{2} \sin (\Omega t-\phi)
\end{gathered}
$$

3. Time-Dependent-Only Axial Load Simplification. In this case the equation of motion and the discretized equation are

$$
\begin{gathered}
\varepsilon\left(v_{, t t}+\frac{1}{\pi^{4}} v_{, x x x x}-m_{s} a \Omega^{2} \cos \Omega t v_{, x x}\right) \\
=(1-x) a \Omega^{2} \sin \Omega t \\
\quad+\frac{a^{2}}{2} \Omega^{2} \sin 2 \Omega t \\
\varepsilon\left(\ddot{g}+m_{s} a \Omega^{2} \pi^{2} \cos \Omega t g+g\right)=\frac{2 a \Omega^{2}}{\pi}(\sin \Omega t+a \sin 2 \Omega t)
\end{gathered}
$$

Runge-Kutta Simulation. Figure 5 shows the response $g$ of the connecting rod at $\Omega=0.8$. The other parameters are the same as in the low crank speed case. Line (1) represents the response from the Lagrangian strain formulation. Line (2) is from linear strain simplification. Lines (3) and (4) are from Eqs. (38) and (40), respectively. At this high speed the response from the three simplified formulations are almost ten times larger than the one predicted from Lagrangian strain formulation. Figure 6 shows the response $f$ from the Lagrangian strain and linear strain formulations.

While the three simplified formulations fail to reproduce the results obtained from Lagrangian strain formulation, it is possible to simplify Eqs. (35) and (36) directly by neglecting the higher order terms. By ignoring the $\varepsilon^{2}$ - and $\varepsilon$-terms in Eq. (35) and assuming $a$ to be small, we can express $f$ in terms of $g$ as

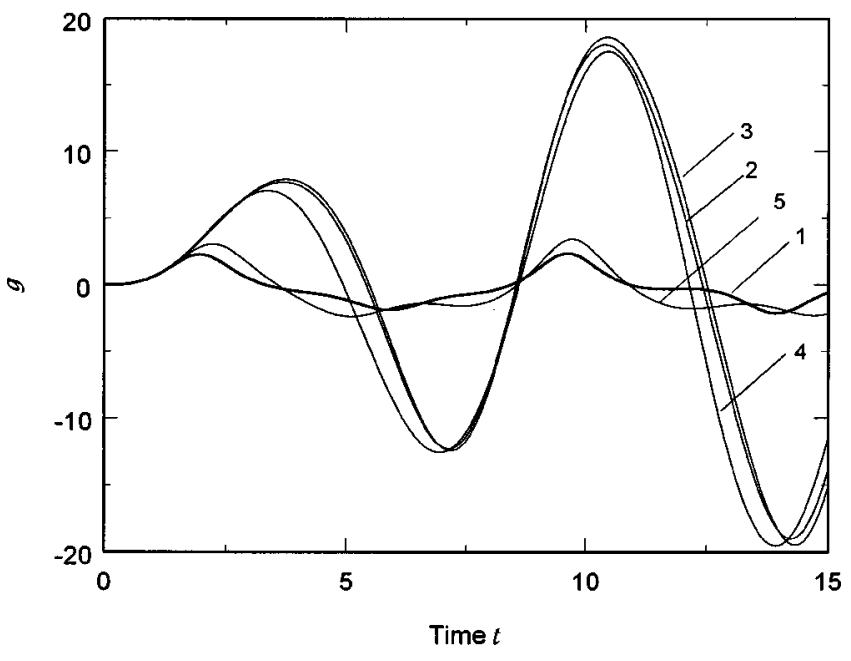

Fig. 5 Response $g$ of the connecting rod at $\Omega=0.8$. (1) Lagrangian strain formulation. (2) Linear strain formulation. (3) Axial load by integrating axial equilibrium equation. (4) Timedependent-only axial load formulation. (5) Response predicted by Eq. (42). 


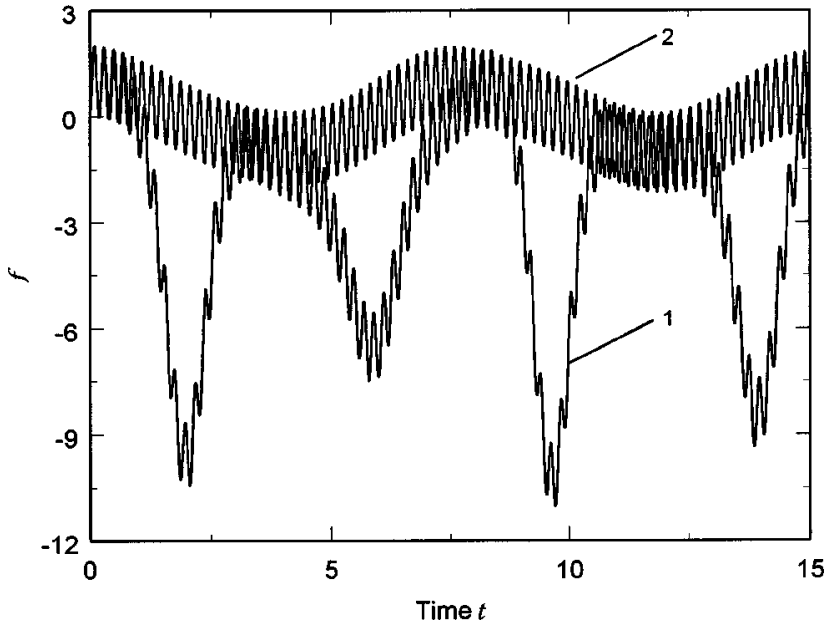

Fig. 6 Response $f$ of the connecting rod at $\Omega=0.8$. (1) Lagrangian strain formulation. (2) Linear strain formulation.

$$
f=-\frac{28}{15} g^{2}+8 a \pi \Omega^{2}\left(\pi m_{s}+2\right) \cos \Omega t
$$

After substituting Eq. (41) into Eq. (36), we obtain the single equation for $g$,

$$
\begin{gathered}
\varepsilon\left\{\ddot{g}+g+\frac{112}{15} a \Omega^{2} \cos \Omega t\left(m_{s}+\frac{2}{\pi}\right) g+\left(\frac{3}{8}-\frac{392}{225 \pi^{2}}\right) g^{3}\right\} \\
=\frac{2 a \Omega^{2}}{\pi}(\sin \Omega t+a \sin 2 \Omega t)
\end{gathered}
$$

The response from Eq. (42) is plotted in Fig. 5 as line (5), which approximates line (1) much better than those from other simplifications. The main difference between Eq. (42) and those from the simplified formulations is the cubic term. If this cubic term is ignored, Eq. (42) will return to the inhomogeneous Mathieu equation. Since this cubic term is also a dominant term, it will have a substantial effect on the stability property of the Mathieu equation. It is noted that if $a$ approaches zero, on the other hand, Eq. (42) will become a Duffing equation [18].

\section{Accuracy of One-Mode Approximation}

Up to this point we have assumed that the one-mode approximation can well represent the essence of responses $u$ and $v$. While this is usually the case from previous experience, it is desirable to check the accuracy of this assumption. For more accurate approximation we can assume multiple-mode expansion as

$$
\begin{gathered}
u(x, t)=\sum_{n=1}^{N} f_{n}(t) \sin \frac{(2 n-1) \pi x}{2} \\
v(x, t)=\sum_{n=1}^{N} g_{n}(t) \sin n \pi x
\end{gathered}
$$

where $N$ is the number of modes used in the expansion. After substituting Eqs. (43) and (44) into Eqs. (32) and (33), multiplying Eqs. (32) and (33) by $\sin [(2 n-1) \pi x / 2]$ and $\sin n \pi x$, respectively, and integrating by parts from $x=0$ to 1 we obtain $2 N$ equations for $f_{n}$ and $g_{n}$. Runge-Kutta method can then be used to calculate the solution. For a two-mode approximation $N=2$, lines (2) and (3) in Fig. 7 represent $g_{1}$ and $g_{2}$, respectively when $\Omega=0.8$. From the observation that $g_{2}$ is much smaller than $g_{1}$ in magnitude, we confirm that the first mode is much more important than the second one in approximating the solution $v$. The solution $g$ from the one-mode approximation by solving Eqs. (16) and (17) is also plotted in Fig. 7 as line (1) for comparison. Since line (1)

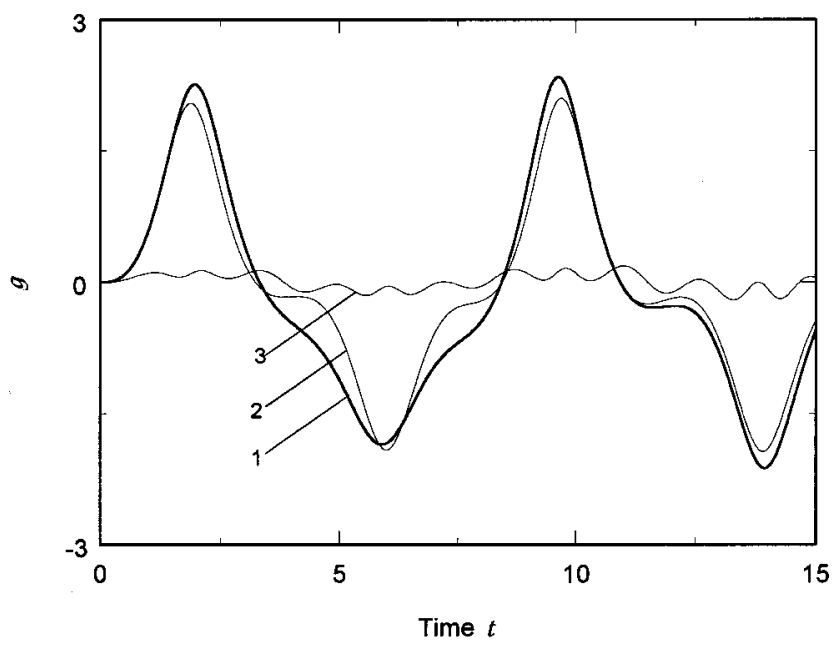

Fig. 7 Comparison of one-mode and two-mode approximations at $\Omega=0.8$. (1) $g$ from one-mode approximation. (2) $g_{1}$ and (3) $g_{2}$ from two-mode approximation.

is fairly close to line (2), we conclude that the one-mode approximation can indeed retain the essence of the solutions $u$ and $v$.

\section{Conclusions}

In this paper we point out for the first time that the Lagrangian strain formulation and the axial constitutive relation are required in calculating the response of a flexible connecting rod with crank speed comparable to the first bending natural frequency of the connecting rod. The conventional approach by assuming either the axial load is dependent on time only or is proportional to the linear axial strain gives satisfactory result only when the crank speed is low.

The first potential impact of this conclusion is that this could be the general case in all flexible mechanisms. Therefore, in developing design software for dynamic analysis of general flexible mechanisms based on more powerful technique such as finite element method, it is crucial to retain the coupling effect between the axial and the transverse vibrations. Secondly, as pointed out in Eq. (42) the cubic term from Lagrangian strain formulation may have a substantial effect on the parametric instability of the Mathieu equation obtained from conventional simplifications. Therefore, the critical crank speed predicted by the conventional formulation $[7,12]$ may need a detailed examination. These two possible research directions should be undertaken in the future in order for us to better understand the dynamic response of flexible mechanisms operating at high speed.

\section{Acknowledgment}

The results presented here were obtained in the course of research supported by a grant from the National Science Council of the Republic of China.

\section{References}

[1] Midha, A., Erdman, A. G., and Frohrib, D. A., 1978, "Finite Element Approach to Mathematical Modeling of High Speed Elastic Linkages," Mech. Mach. Theory, 13, pp. 603-618.

[2] Shabana, A. A., 1989, Dynamics of Multibody Systems, John Wiley and Sons, New York.

[3] Nagarajan, S., and Turcic, D. A., 1990, "General Methods of Determining Stability and Critical Speeds for Elastic Mechanism Systems," Mech. Mach. Theory, 25, No. 2, pp. 209-223.

[4] Neubauer, A. H., Cohen, R., and Hall, A. S., 1966, "An Analytical Study of the Dynamics of an Elastic Linkage," ASME J. Eng. Ind., 88, pp. 311-317.

[5] Badlani, M., and Midha, A., 1982, "Member Initial Curvature Effects on the Elastic Slider-Crank Mechanism Response," ASME J. Mech. Des., 104, pp. 159-167.

[6] Badlani, M., and Midha, A., 1983, "Effect of Internal Material Damping on 
the Dynamics of a Slider-Crank Mechanism,” ASME J. Mech., Transm., Autom. Des., 105, pp. 452-459.

[7] Badlani, M., and Kleninhenz, W., 1979, "Dynamic Stability of Elastic Mechanism,” ASME J. Mech. Des., 101, pp. 149-153.

[8] Tadjbakhsh, I. G., 1982, "Stability of Motion of Elastic Planar Linkages With Application to Slider Crank Mechanism," ASME J. Mech. Des., 104, pp. 698 703.

[9] Tadjbakhsh, I. G., and Younis, C. J., 1986, "Dynamic Stability of the Flexible Connecting Rod of a Slider Crank Mechanism," ASME J. Mech., Transm., Autom. Des., 108, pp. 487-496.

[10] Zhu, Z. G., and Chen, Y., 1983, "The Stability of the Motion of a Connecting Rod,” ASME J. Mech., Transm., Autom. Des., 105, pp. 637-640.

[11] Viscomi, B. V., and Ayre, R. S., 1971, "Nonlinear Dynamic Response of Elastic Slider-Crank Mechanism,” ASME J. Eng. Ind., 93, pp. 251-262.

[12] Hsieh, S. R., and Shaw, S. W., 1994, "The Dynamic Stability and Nonlinear Resonance of a Flexible Connecting Rod: Single Mode Model," J. Sound Vib., 170, pp. 25-49.
[13] Jasinski, P. W., Lee, H. C., and Sandor, G. N., 1970, "Stability and SteadyState Vibrations in a High Speed Slider-Crank Mechanism," ASME J. Appl. Mech., 37, pp. 1069-1076.

[14] Jasinski, P. W., Lee, H. C., and Sandor, G. N., 1971, "Vibrations of Elastic Connecting Rod of a High Speed Slider-Crank Mechanism," ASME J. Eng. Ind., 93, pp. 636-644.

[15] Chu, S. C., and Pan, K. C., 1975, "Dynamic Response of a High Speed Slider-Crank Mechanism With an Elastic Connecting Rod," ASME J. Eng. Ind., 97, pp. 542-550.

[16] Fung, R.-F., and Chen, H.-H., 1997, "Steady-State Response of the Flexible Connecting Rod of a Slider-Crank Mechanism With Time-Dependent Boundary Condition," J. Sound Vib., 199, pp. 237-251.

[17] Bolotin, V. V., 1964, The Dynamic Stability of Elastic Systems, Holden-Day, San Francisco.

[18] Nayfeh, A. H., and Mook, D. T., 1979, Nonlinear Oscillations, Wiley, New York 